\title{
The effect of neoadjuvant chemotherapy on pathological response and the hormone receptor profile in locally advanced breast carcinomas
}

\author{
BS Subhendu, ${ }^{1}$ R Shekhar ${ }^{2}$ \\ ${ }^{1}$ Associate Professor, Department of General Surgery, Bankura Sammilani Medical College, Bandura, West Bengal, India \\ ${ }^{2}$ Post Graduate Trainee, Department of General Surgery, Bankura Sammilani Medical College, Bandura, West Bengal, India
}

Corresponding author: Subhendu Bikas Saha (sunsubg@gmail.com)

\begin{abstract}
Background: The impact of neoadjuvant chemotherapy (NACT) on tumour biomarkers and the histopathological response to treatment in breast cancer specimens remains controversial. Chemotherapy and hormonal therapy decisions for breast cancer management are influenced by the expression of tumour biomarkers: estrogen receptor (ER), progesterone receptor (PR) and human epidermal growth receptor 2 (HER2). On the other hand, pathological response is an indicator of chemotherapy effectiveness. The study of the effect of NACT in breast cancer is an important issue.

Objectives: To assess the changes to biological markers ER, PR, and HER2, and the pathological response in locally advanced breast cancer patients after neoadjuvant chemotherapy.

Methods: 100 patients with locally advanced breast cancer were assessed with core needle biopsy for biological markers (ER, PR, HER2) and pathological grading. Subsequently they were treated with six cycles of taxane based NACT followed by surgical resection. Biological markers and the pathological response (assessed by the Miller Payne grading system) were re-evaluated to assess changes.

Results: The patient mean age was $45.62 \pm 7.12$ years. Most patients $(56 \%)$ were postmenopausal. Clinical disease stage ranged between any T N2, T3 N1-2, T4 N0-2. Post NACT, pathological complete response rate was $14 \%$, ER positivity decreased from $80(80 \%)$ to $78(78 \%)(p=0.67)$. PR positive dropped from 66 to $62 \%(p=0.002)$, and HER2 receptor positivity was increased from $22 \%$ to $28 \%$ (p-value 0.000 ).

Conclusion: It was observed that biological markers (ER, PR, HER2) and the histopathological response of breast cancer may change after NACT. This change may affect treatment decisions.
\end{abstract}

S Afr J Surg 2018;56(4)

http://dx.doi.org/10.17159/2078-5151/2018/v56n4a2609

\section{Background}

Breast cancer is the most common site specific cancer in women and is the leading cause of death from cancer for women aged 20-59 years. ${ }^{1}$ The current NCCN recommendations for treatment of operable advanced loco-regional breast cancer are neoadjuvant chemotherapy with an anthracycline or taxane containing regimen or both, followed by mastectomy or lumpectomy with axillary lymph node dissection, and, if necessary, followed by adjuvant therapy. ${ }^{2}$ The decision for adjuvant therapy is strongly influenced by tumour biomarkers including (ER), (PR) and HER2 that are performed on core needle biopsy specimens using Immunohistochemical (IHC) staining and/or Fluorescence in situ hybridization (FISH) for HER2 testing. After NACT and surgery, biomarker studies are repeated on resected specimens and may be different from previous biomarker studies performed on core needle biopsy. ${ }^{3}$ This may be due to chemotherapy effect or intratumoral heterogeneity and these changes may alter treatment decisions following surgery. After NACT the pathological response to treatment is assessed. Patients whose tumours had pathological complete response with NACT have statistically improved survival outcomes compared to those patients whose tumours demonstrated only a partial response, remained stable or progressed on treatment. ${ }^{4}$ In this study we aimed to assess the effect of NACT on LABC with respect to the tumour biomarker profile and histopathological response grade.

\section{Materials and methods}

During the period February 2016 to August 2017, 100 confirmed untreated cases of LABC attending our institution 
(Bankura Sammilani Medical College) were included in the study and analysed prospectively. Confirmation was done by triple assessment and standard metastatic workup. Tumour markers were performed on core needle biopsy specimens which were preserved for further pathological analysis (ER, PR and HER2 status and pre-chemotherapy tumour cellularity). Patients were treated with taxane based NACT shortly after diagnosis (all patients received 6 cycles of cyclophosphamide, doxorubicin, paclitaxel that is TAC, $\mathrm{T}=$ taxane, $\mathrm{A}=$ Adriamycin, $\mathrm{C}=$ cyclophosphamide). Surgery was performed 2 weeks after the last cycle of NACT. ER, PR and, HER2 receptor status was assessed using standard IHC techniques on both pre-NACT and post-surgery specimens (Herceptin was given according to post-chemotherapy HER2 status). For ER and PR nuclear expression in $>1 \%$ tumour cells was accepted as positive and $3+$ was considered as HER2 positive ("Fluorescence in situ hybridization (FISH) is not available at our institution. HER2 was only assessed by immunohistochemical methods. We could therefore not evaluate HER $22^{+}$cases, as assessed by immunohistochemical method, further. Only HER $3^{+}$cases were considered positive. HER2 $1^{+}$and HER2 $2^{+}$were considered negative.")

Pathological response was assessed by the Miller Payne grading system in the 2 previously mentioned specimens for all patients. ${ }^{5}$ All specimens were concurrently reviewed by two pathologists.

Data were entered in Micro Soft (MS) excel spread sheet. Quantitative and the qualitative data were described by estimating mean, standard deviation (SD) and proportion, respectively. Data display was done using tables and charts. McNemar Chi-square $\left(\chi^{2}\right)$ test was used to draw the inference about outcome variables. A p-value $\leq 0.05$ with $95 \%$ confidence interval $(\mathrm{CI})$ at $5 \%$ precision was considered significant. MS excel and statistical software SPSS 22.2.0 version were utilised for the purpose of data analysis.

\section{Results}

The average age of the patients was $45.62 \pm 7.12$ (mean $\pm \mathrm{SD}$ ) years, range $36-60$ years. The majority of the patients $(56 \%)$ were postmenopausal. The predominant histological subtype was infiltrating ductal carcinoma (96\%) others being medullary (2\%) and mucinous carcinomas (2\%).

ER positivity dropped from 80 to $78 \%$ following NACT. There was a corresponding increase of ER negativity from 20 to 22 . $(p=0.671)$.

Sixty-six $(66 \%)$ patients were initially PR positive and thirty-four (34\%) patients were PR negative. After NACT, PR negative cases increased to 38 . This change was statistically significant $(\mathrm{p}=0.002)$. Sixty-six $(66 \%)$ patients were initially PR positive and thirty-four (34\%) patients were PR negative. After NACT, PR negative cases increased to 38 . This change was statistically significant $(\mathrm{p}=0.002)$. HER2 receptor positivity was increased after chemotherapy from $22-28 \%$. The change of HER2 receptor expression was also statistically significant $(\mathrm{p}=0.000)$ (Table1).

The Miller-Payne Grading System (Table 2) was applied for the assessment of post-NACT pathological response. Fourteen

Table 1: Change of ER status before and after chemotherapy $(n=100)$

\begin{tabular}{lcccc}
\hline ER status before chemo & \multicolumn{2}{c}{ ER status after chemo } & Total & p value at df 1 \\
\cline { 2 - 4 } & \multicolumn{2}{c}{ Positive (\%) } & Negative (\%) & \\
\hline Positive (\%) & $64(80)$ & $16(20)$ & $80(80)$ \\
Negative (\%) & $14(70)$ & $6(30)$ & $20(20)$ & 0.671 \\
Total & $78(78)$ & $22(22)$ & $100(100)$ \\
Comment: Before chemotherapy $80 \%$ patients was ER positive and after chemo it became $78 \%$ but the result was statistically insignificant $\mathrm{p}>0.05)$.
\end{tabular}

Table 2: Change of PR status before and after chemotherapy $(n=100)$

\begin{tabular}{|c|c|c|c|c|}
\hline \multirow[t]{2}{*}{ PR status before chemo } & \multicolumn{2}{|c|}{ PR status after chemo } & \multirow[t]{2}{*}{ Total (\%) } & \multirow[t]{2}{*}{$p$ value at df 1} \\
\hline & Positive (\%) & Negative (\%) & & \\
\hline Positive (\%) & $52(79)$ & $14(21)$ & $66(66)$ & \\
\hline Negative (\%) & $10(29)$ & $24(71)$ & $34(34)$ & 0.002 \\
\hline Total (\%) & $62(62)$ & $38(38)$ & $100(100)$ & \\
\hline
\end{tabular}

Table 3: Change of Her-2/neu receptor status before and after chemotherapy $(n=100)$

\begin{tabular}{|c|c|c|c|c|}
\hline \multirow[t]{2}{*}{ Her-2/neu before chemo } & \multicolumn{2}{|c|}{ Her-2/neu after chemo } & \multirow[t]{2}{*}{ Total (\%) } & \multirow[t]{2}{*}{$p$ value at df 1} \\
\hline & Positive (\%) & Negative (\%) & & \\
\hline Positive (\%) & $18(82)$ & $4(18)$ & $22(22)$ & \\
\hline Negative (\%) & $10(13)$ & $68(87)$ & $78(78)$ & 0.00 \\
\hline Total (\%) & $28(28)$ & $72(72)$ & $100(100)$ & \\
\hline
\end{tabular}


percent $(14 \%)$ were grade 5 , having pathological complete response, the majority $(57 \%)$ had a triple negative hormone receptor status (Figure 1).

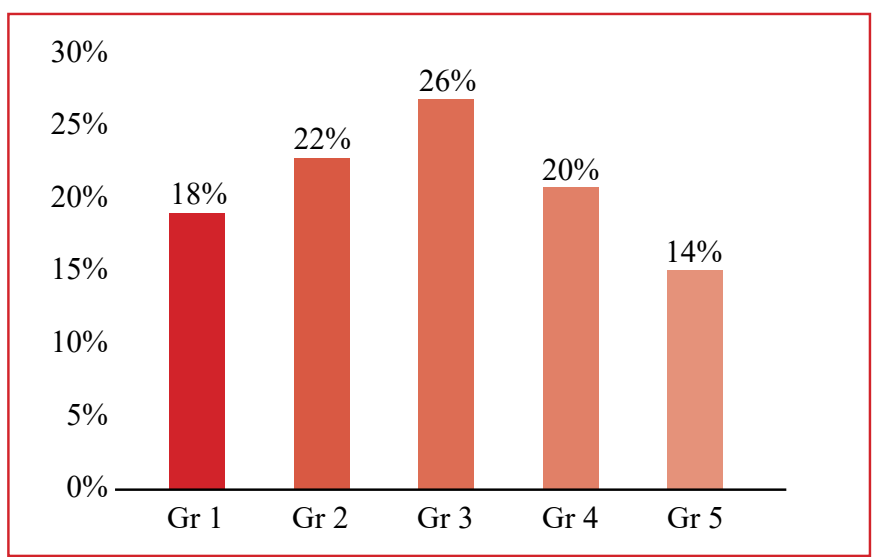

Fig. 1: Distribution of patients according to pathological response grading $(n=50)$

Comment: After chemotherapy $14 \%$ patients were in grade $5,20 \%$ in grade 4 , $26 \%$ in grade $3,22 \%$ in grade 2 and $18 \%$ in grade 1 of pathological response.

\section{Discussion}

The fact that chemotherapeutic agents cause some changes on some components of the tumour cells has been known since the 1960s. First, Waller demonstrated changes such as enlargement of the nucleus, swelling of the cytoplasm, vacuolization in the cytoplasm/nucleus in tumour cells following systemic administration of busulphan. ${ }^{6}$ Since changes in the molecular properties of cancer cells may affect the tumour behavior and therefore the treatment plan, studies investigating how the chemotherapeutic agents affect tumour grade, receptor properties of tumour cells and histopathological characteristics have recently been increasing in number. In this study we examined the quantitative changes in ER, PR and HER 2 and the histopathological response in breast cancer patients receiving NACT with doxorubicin, cyclophosphamide and paclitaxel.

\section{Effect of NACT on Estrogen receptor and Progesterone receptor}

Several studies have looked at hormone receptor changes in tumour cells with neoadjuvant chemotherapy. Taucher et al. studied the effect of neoadjuvant therapy in a group of 214 patients and reported that $14 \%$ of the tumours which were ER positive and $51 \%$ of the tumours which were PR positive initially became hormone receptor negative and both changes were statistically significant $(p=0.02$ for ER and $p=0.0005$ for PR). ${ }^{7}$ In a study by Makris et al. 11 patients showed a statistically significant change in ER status $(p=0.04)$ and 15 patients showed a change in PR status which was not statistically significant. ${ }^{8}$ Dede et al. reviewed 35 patients and showed that $5.7 \%$ of patients changed from ER positive to negative; $21.1 \%$ became PR negative and $9 \%$ became PR positive after NACT. However, changes in ER and PR status were not statistically significant $(\mathrm{p}=0.500$ and $\mathrm{p}=0.549$, respectively). ${ }^{9}$ Some investigators support the idea that possible changes caused by neoadjuvant therapy in hormone receptor status of the tumour do not show significant importance..$^{10}$

In our study, 80 patients $(80 \%)$ were initially ER positive. Following NACT this dropped by $2 \%$, which was statistically insignificant.

The PR positivity changed from $66(66 \%)$ to 62 which was statistically significant.

\section{Effect of NACT on HER2 status}

The variations of the HER2 biological marker in the primary tumour and its synchronous/metachronous metastasis and changes in HER 2 status after neoadjuvant treatment have been assessed in several studies. ${ }^{11}$ Studies examining the effect of NACT on HER 2-expression report conflicting results. Adams et al. reported increased expression of HER 2 after NACT in breast cancer patients in whom the hormone receptor status remained unchanged. ${ }^{12}$

Taucher et al. evaluated HER 2 changes with immunohistochemical methods in addition to hormone receptor changes in their study. ${ }^{7}$ They showed that HER 2 positivity changed after NACT but the results were not statistically significant. They also reported that confirmation of the results with FISH method revealed that the difference was much smaller compared with immunohistochemistry. Another study by Burcombe et al. showed a change in HER 2 expression in 9 of 118 patients with NACT (from $3+$ to $2+$ in 3 patients and from $2+$ to $3+$ in 5 patients (done by immunohistochemical method) and the authors suggested reevaluation of HER 2 in the residual tumour in patients whose initial HER 2 studies showed mild to high positivity after neoadjuvant therapy. ${ }^{13}$ In addition, Neubauer et al. reported $13 \%$ of the tumours switched from HER 2 positive to negative after neoadjuvant therapy. ${ }^{14}$ In a study conducted by Dede et al. on 36 patients, HER 2 expression remained unchanged in 30 patients $(83 \%)$ while 6 patients $(16.6 \%)$ showed some type of change: 3 converted from positive to negative and 3 became negative to positive. On the other hand, several studies report no change in HER 2 expression with neoadjuvant therapy. ${ }^{9}$

In the present study baseline, HER2 positivity was found among $22(22 \%)$ patients and the rest, i.e. $78(78 \%)$ were revealed to be HER2 negative. However, after six cycles of NACT this figure became $28(28 \%)$ and $72(72 \%)$, respectively. Further analysis reflected that four out of 22 previously HER2 positive patients became negative after NACT whereas 10 out of 78 previously HER2 negative patients became positive after the therapy. It meant that 19 positive patients remained positive and 68 negative patients continued to have negative HER2 status in spite of getting NACT. This change in respect of the HER2 receptor expression was revealed to be statistically significant $\left[\mathrm{p}=0.000\right.$ as per McNemar $\chi^{2}$ test] (Table 1).

\section{Effect of NACT on pathological response}

Several studies have looked at pathological response following 
NACT. In one study, 88 patients with operable breast cancer treated with NACT were analysed. Histological response was assessed according to the_Miller-Payne grading (MPG) and Residual Disease in Breast and Nodes (RDBN). Ten patients $(11.4 \%)$ achieved a pathological complete response according to RDBN and $13.6 \%$ according to MPG. Patients with triple negative breast cancer were more likely to get pathological complete response. ${ }^{15}$ Rodenhuis et al. studied the neoadjuvant response in 267 patients: 55 patients (21\%) had a pathological complete response in both breast and axilla. Pathological complete responses were more frequently seen in patients with triple negative tumor. ${ }^{16}$ Elghazaly et al. studied 125 patients with stage-II and stage-III non-inflammatory breast cancer treated with six cycles chemotherapy. Among them $20 \%$ of patients achieved pathological complete response. ${ }^{17}$

In our study $14(14 \%)$ of patients had pathological complete response following NACT. The majority $(57 \%)$ of these had triple negative hormone receptor status.

\section{Conclusion:}

After treatment with NACT the pathological response grade of the breast cancer varies according to biomarker status, with triple negative breast cancers attaining the highest rate of a pathological complete response. The hormonal profile and HER2 receptor status may change to some extent. Proper pathological assessment and immunohistochemistry should be done before and after NACT to guide the surgeon in formulating appropriate treatment plans.

\section{Conflict of interest}

The authors declare that they have no conflict of interest.

\section{REFERENCES}

1. Siegel R, Naishadham D, Jemel A. Cancer statistics, 2013. CA Cancer J Clin. 2013;63:11-30.

2. 0044 J Natl Compr Canc Netw. 2017;15:433-451. doi: 10.6004/ jncen.2017

3. Jin G, Han Y, Liu C, Chen L, Ding B, Xuan S, et al. Evaluation of biomarker changes after administration of various neoadjuvant chemotherapies in breast cancer. Int J Clin Exp Pathol. 2015; 8:914-21.

4. Kuerer HM, Newman LA, Smith TL, et al. Clinical Course of breast cancer patients with complete pathologic primary tumor and axillary lymph node response to doxorubicin-based neoadjuvant chemotherapy. J Clin Oncol. 1999;17:460-9.

5. Ogston KN, Miller ID, Payne S, et al. A new histological grading system to assess response of breast cancers to primary chemotherapy; prognostic significance and survival. Breast. 2003;12:320-7. [PubMed]

6. Waller U. Giant nuclei after myleran therapy and splenic irradiation in chronic myeloid leukemia. Pathol Microbiol (Basel). 1960;23:283-90.

7. Taucher S, Rudas M, Gnant M, et al. Sequential steroid hormone receptor measurements in primary breast cancer with and without intervening primary chemotherapy. Endocr Relat Cancer. 2003;10:91-8.

8. Makris A, Powels TJ, Allred DC, et al. Quantitative changes in cytological molecular markers during primary medical treatment of breast cancer: A pilot study. Breast Can Res Treat. 1999;53:51-9.

9. Dede DS, Gumuskaya B, et al. Evaluation of changes in biologic markers ER, PR, HER 2 and Ki-67 index in breast cancer with administration of neoadjuvant dose dense doxorubicin, cyclophosphamide followed by paclitaxel chemotherapy. JBUON. 2013;18(2):366-71.

10. Bottini A, Berruti A, Bersiga A, et al. Relationship between tumour shrinkage and reduction in Ki-67 expression after primary chemotherapy in human breast cancer. Br J Cancer. 2001;85:1106-12.

11. Arapantoni-Dadioti $\mathrm{P}$, Valvanis $\mathrm{C}$, Gavressea $\mathrm{T}$, et al. Discordant expression of hormone receptors and HER2 in breast cancer. A retrospective comparison of primary tumors with paired metachronous recurrences or metastases. J BUON. 2012;17:277-83.

12. Adams AL, Eltoum I, Krontiras H. The Effect of Neoadjuvant Chemotherapy on Histologic Grade, Hormone Receptor Status, and Her2/neu Status in Breast Carcinoma. Breast J. 2008;14: 141-6.

13. Burcombe RJ, Makris A, Richman PI, et al. Evaluation of Ki67 proliferation index before and during neoadjuvant chemotherapy for primary breast cancer. Breast Cancer Res Treat. 2002;76: abstr no. 229.

14. Neubauer H, Gall C, Vogel $U$, et al. Changes in tumour biological markers during primary systemic chemotherapy. Anticancer Res, 2008; 28:1797-1804.

15. Zhao Y, Dong X, Li R, Ma X, Song J, Li Y, Zhang D. Evaluation of the pathological response and prognosis following neoadjuvant chemotherapy in molecular subtypes of breast cancer. Onco Targets Ther. 19 Jun 2015;8:1511-21. doi: 10.2147/OTT.S83243. eCollection 2015

16. Rodenhuis S, Richel DJ, van der WE, Schornagel JH, Baars JW, Koning CC, et al. Randomised trial of high-dose chemotherapy and haemopoietic progenitor-cell support in operable breast cancer with extensive axillary lymph-node involvement (see comments) Lancet. 1998;352:515-21. [PubMed]

17. Elghazaly H, Razek NA, Anies E, Elia S, Youssef O. Correlation of Pathological Complete Response with Radiological Evaluation after Neoadjuvant Chemotherapy of Breast Carcinoma. J Cell Sci Ther. 2013;4:149. doi: 10.4172/21577013.1000149 